\title{
Breakup of an Elongated Droplet in a Centrifugal Field ${ }^{\dagger}$
}

\author{
Els H. A. de Hoog and Henk N. W. Lekkerkerker* \\ Van 't Hoff Laboratory for Physical and Colloid Chemistry, Debye Institute, Utrecht University, \\ Padualaan 8, 3584 CH Utrecht, The Netherlands
}

Received: April 9, 2001; In Final Form: July 23, 2001

\begin{abstract}
The breakup of an elongated drop in the presence of a centrifugal field was studied. The system used was a phase-separated colloid-polymer suspension; the elongated drop consisted of the lighter polymer-rich fluid phase and the surrounding fluid was its coexisting colloid-rich fluid phase. We found that the growth rate of the fastest growing disturbance of the drop, which eventually leads to its breakup, is decreasing upon an increase of the rotational speed, whereas the wavelength of the disturbance is increasing upon an increase of the rotational speed. We present a simple analysis of the effect of the centrifugal field which accounts quantitatively for these features. Furthermore, this analysis allows for the determination of the interfacial tension from the measured growth rates.
\end{abstract}

\section{Introduction}

Dynamics of drop deformation and breakup in viscous fluids have been experimentally, numerically, and theoretically investigated. ${ }^{1}$ Many of the research problems described in the droplet breakup literature are (partly) motivated by industrial processes. Important applications can be found in blending of polymers, ${ }^{2}$ in ink-jet printers, ${ }^{3}$ in emulsion formation and rheology, ${ }^{4}$ and in the measurement of interfacial tension from time-dependent shape changes. 5,6 Our interest in droplet breakup dynamics lies in the possibility of determining the interfacial tension between coexisting colloidal fluids from it.

The interfacial tension in a colloid-polymer suspension, phase separated into a colloidal-gas phase and a colloidal-liquid phase, is very low, on the order of $1-10 \mu \mathrm{N} / \mathrm{m}$ and has recently been studied experimentally ${ }^{7-9}$ and theoretically. ${ }^{10-12}$

In the experiments, the interfacial tension was measured with the spinning drop method, which is appropriate to measure such low interfacial tensions. In this method a small droplet of the lighter phase is injected into a tube filled with the denser phase. The tube is rotated and the droplet is elongated along the rotational axis, which is perpendicular to the gravitational field. The shape of the droplet is determined by the centrifugal forces and the interfacial tension. In 1942 Vonnegut $^{13}$ derived an approximate equation to determine the interfacial tension from the dimensions of the droplet:

$$
\gamma=\frac{\omega^{2} R^{3} \Delta \rho}{4}
$$

with $\gamma$ the interfacial tension, $\omega$ the rotational speed, $R$ the radius of the droplet, and $\Delta \rho$ the density difference between the two phases. Princen et al. ${ }^{14}$ derived an extension of this equation in 1967 , which is also valid when the length of the drop is smaller than 4 times the diameter.

It was observed that the elongated droplet that is produced in the spinning drop experiment can break up into several smaller droplets by a decrease in the rotational speed. ${ }^{8}$ In these

$\doteqdot$ Part of the special issue "Howard Reiss Festschrift".

* Corresponding author. E-mail: H.N.W.Lekkerkerker@chem.uu.nl.

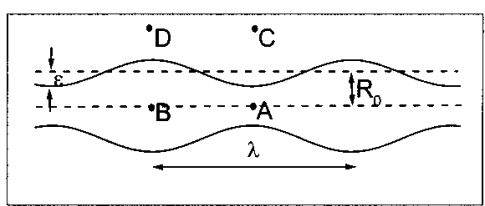

Figure 1. Schematic representation of a breaking droplet.

previous experiments it was used to obtain several smaller droplets from a larger one. In 1878, Rayleigh ${ }^{15}$ treated the instability of a long, thin, cylindrical liquid thread, and this work was extended by Tomotika ${ }^{16}$ in 1935 . Later, Rumscheidt and Mason ${ }^{17}$ realized that this theory could be used for determination of the interfacial tension. This method is also referred to as the breaking thread method, and it has been applied to, for example, oils ${ }^{5}$ and polymers..$^{5,6}$

Consider a long liquid cylinder with radius $R_{0}$ and viscosity $\eta_{1}$ surrounded by another liquid with viscosity $\eta_{2}$ (see Figure 1). A deformation

$$
R(z)=R_{0}+\epsilon \sin \frac{2 \pi z}{\lambda}
$$

with a wavelength $\lambda$ larger than the original circumference of the drop, $\lambda \geq 2 \pi R_{0}$, will grow and eventually cause the drop to break. Here $R$ is the radius of the droplet, $R_{0}$ is the intial radius of the liquid thread, $\epsilon$ is the amplitude of the disturbance, and the $z$-direction is parallel to the axis of rotation. The breakup of an elongated droplet into smaller ones is driven by a decrease of the total interface between the two fluids. From the work of Tomotika ${ }^{16}$ it follows that the amplitude $\epsilon$ of the disturbance grows exponentially with time $t$.

$$
\epsilon=\epsilon_{0} \exp (q t)
$$

The growth rate $q$ of a disturbance is given by

$$
q=\frac{\gamma\left(1-X^{2}\right) \Phi\left(\eta_{1} / \eta_{2}, X\right)}{2 \eta_{2} R_{0}}
$$

where $X$ is a dimensionless parameter, $X=2 \pi R_{0} / \lambda ; \Phi$ is a function of $X$ and the viscosity ratio of the thread $\left(\eta_{1}\right)$ to the 


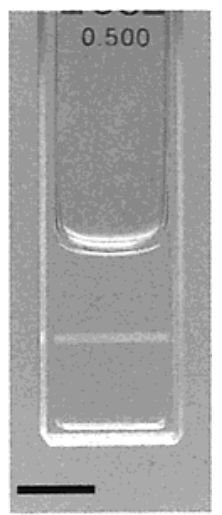

Figure 2. Photograph of the phase-separated colloid-polymer suspension. The tube is tilted over a small angle in order to observe the interface. The bar represents $5 \mathrm{~mm}$.

surrounding fluid $\left(\eta_{2}\right)$. The fastest growing wavelength will be dominant and is determined by the interfacial tension $\gamma$ and the viscosities of both phases. The interfacial tension can now be determined by measuring the growth rate, $q_{\mathrm{m}}$, which is associated with the dominant wavelength, $\lambda_{\mathrm{m}}$.

This breaking thread method was used to measure the interfacial tension by the determination of the breakup growth rate of an elongated liquid drop in the absence of an overall flow field. In our previous experiments, ${ }^{8}$ however, the final rotational speed could not be reduced to zero as it was needed to balance the gravitational force on the droplet. It was observed that the breakup velocity depends also on the final rotational speed. In this study we investigate the dependence of the breakup velocity on the centrifugal field, expressed by the final rotational speed.

\section{Experimental Section}

A dispersion of silica particles and poly(dimethylsiloxane) (PDMS) polymers in the solvent cyclohexane was used. The silica particles are coated with 1-octadecanol to provide a steric stabilization; the radius of the coated silica particles is $13 \mathrm{~nm}$ with a polydispersity of $19 \% .^{18}$ The PDMS has a radius of gyration of $14 \mathrm{~nm}$; its molecular weight is $97 \mathrm{~kg} / \mathrm{mol}\left(M_{\mathrm{w}} / M_{\mathrm{n}}\right.$ $=1.9) .{ }^{18}$ As the size ratio of the polymer to colloid is larger than 0.3 , we found a gas-liquid phase transition. The cyclohexane almost matches the index of refraction of both the colloidal particles and the polymer. However, the interface between the coexisting phases could be observed clearly. A photograph of the demixed system is given in Figure 2; the tube is slightly tilted to see the interface better. The density difference between the two phases is determined by measuring the phase diagram and the construction of the associated tie lines, as described elsewhere. ${ }^{8,19}$ For the measurements a sample with a colloid volume fraction of 0.237 and a polymer volume fraction of 1.571 was used. The density difference between the coexisting phases is $242 \mathrm{mg} / \mathrm{mL}$.

A home-built spinning drop tensiometer ${ }^{20}$ was used to measure the evolution of a breaking drop in time. The internal diameter of the spinning drop tube measures $4 \mathrm{~mm}$ and its length is $4 \mathrm{~cm}$. The dense phase was injected with a glass capillary, and the tube was closed with a Teflon stopper on each side; one of the stoppers had a hole through which a small droplet of the lower density phase was injected with a microsyringe. This hole could be closed with a small screw. A droplet of the lower density phase was formed by rotating the tube around its axis horizontally. The rotational speed was measured with an optical sensor and the temperature was fixed at $20{ }^{\circ} \mathrm{C}$ using a
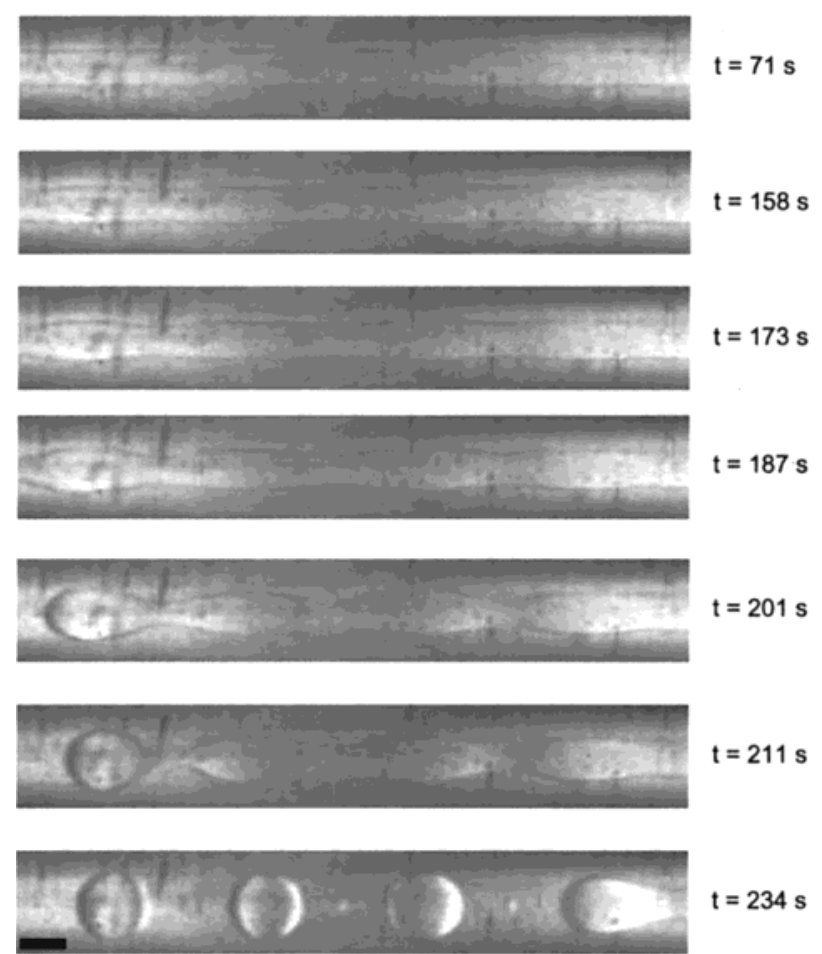

Figure 3. A typical time series of the breakup of the elongated droplet at a final rotational speed of $40 \mathrm{rad} / \mathrm{s}$. The bar represents $0.26 \mathrm{~mm}$, which corresponds to the diameter of the initial liquid thread.
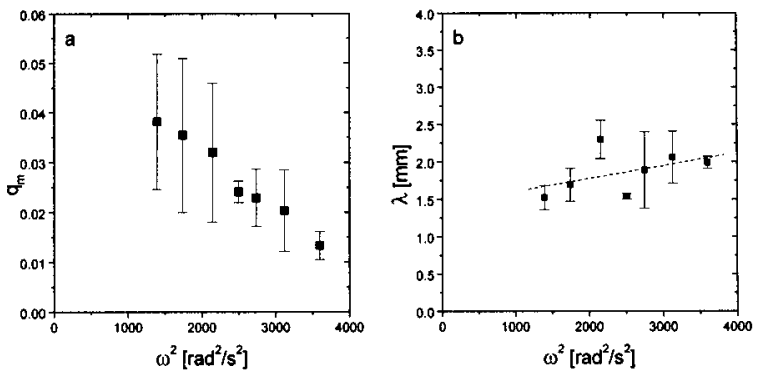

Figure 4. (a) Measured values of the growth rate, $q$, as a function of the rotational speed squared, $\omega^{2}$. (b) Measured values of the wavelength of the distortion, $\lambda$, as a function of the rotational speed squared, $\omega^{2}$.

thermostatic bath connected to the setup. The droplet was elongated for $1 \mathrm{~min}$ at a rotational speed of $500 \mathrm{rad} / \mathrm{s}$. Then, the rotational speed was decreased within $10 \mathrm{~s}$ to a final rotational speed. The breakup process was monitored by taking pictures with a CCD camera at fixed time intervals. A series of experiments was performed in which the final rotational speed was varied between 35 and $70 \mathrm{rad} / \mathrm{s}$. At each final rotational speed the experiment was repeated several times.

\section{Results and Analysis}

The evolution of the droplet in time was followed as a function of the final rotational speed. Only below a final rotational speed of $65 \mathrm{rad} / \mathrm{s}$ was a breakup of the elongated drop observed. A final rotational speed of at least $35 \mathrm{rad} / \mathrm{s}$ was required to balance the gravitational force. Figure 3 shows a typical time series of the breakup.

The growth rate $q$ was determined by the analysis of the diameter of the droplet at necks and bulges. The results are given in Figure 4. Here, the results of two earlier experiments ${ }^{8}$ are also incorporated. We find a decrease in the growth rate with an increase of the final rotational speed (Figure 4a). Furthermore, it was noticed that also the dominant wavelength of the 
disturbance was dependent on the final rotational speed (Figure $4 b)$. The wavelength increases upon an increase of the final rotational speed.

To estimate the dependence of the growth rate on the final rotational speed, first the situation with no centrifugal field is analyzed. Next, the influence of the centrifugal field is incorporated. Finally, we test the developed model using our experimental results, and we will show that the breaking of a droplet can be used to determine the interfacial tension between coexisting fluid phases.

The breakup is driven by pressure difference in the droplet; the pressure in a neck (position A in Figure 1) is higher than that in a bulge (position B in Figure 1) with respect to points $C$ and $\mathrm{D}$, respectively. Points $\mathrm{C}$ and $\mathrm{D}$ are positions in the surrounding fluid phase far from the droplet (see Figure 1). The fluid will flow from point A to point B with a certain velocity, which depends on the magnitude of the pressure difference, the viscosities of both fluids, and the initial radius of the drop. The pressure difference between the two points is calculated using the Laplace equation for the pressure difference between two media due to interfacial tension and separated from each other by a curved interface:

$$
p=\gamma\left(\frac{1}{R_{1}}+\frac{1}{R_{2}}\right)
$$

where the radius in this case can be expressed as a function of the distortion; see eq 2 .

The pressure in point $\mathrm{A}$ with respect to point $\mathrm{C}$ is

$$
\begin{aligned}
p_{\mathrm{A}} & =p_{\mathrm{C}}+\gamma\left[\frac{1}{R_{0}-\epsilon}+\left(-\frac{\mathrm{d}^{2} R}{\mathrm{~d} z^{2}}\right)\right] \\
& =p_{\mathrm{C}}+\frac{\gamma}{R_{0}}\left[1+\frac{\epsilon}{R_{0}}-\epsilon R_{0}\left(\frac{2 \pi}{\lambda}\right)^{2}\right]
\end{aligned}
$$

Similarly, the pressure in point B with respect to point D is

$$
p_{\mathrm{B}}=p_{\mathrm{D}}+\frac{\gamma}{R_{0}}\left[1-\frac{\epsilon}{R_{0}}+\epsilon R_{0}\left(\frac{2 \pi}{\lambda}\right)^{2}\right]
$$

Assuming that the pressure in points $\mathrm{C}$ and $\mathrm{D}$ is equal, the pressure difference (due to the difference in curvature of the interface) is

$$
\Delta p_{\text {Laplace }}=p_{\mathrm{A}}-p_{\mathrm{B}}=\frac{2 \gamma \epsilon}{R_{0}^{2}}\left(1-X^{2}\right)
$$

From this equation the minimum value for the wavelength of the distortion is derived easily. A droplet will break when this pressure difference is larger than zero, which is true for $X$ $\left(=2 \pi R_{0} / \lambda\right)<1$. Therefore, the wavelength should be larger than the circumference of the drop $\left(\lambda>2 \pi R_{0}\right)$.

The evolution of the breakup of the droplet depends on the growth rate times the amplitude of the distortion, as is shown by eq 3 . Furthermore, it can be defined by a mobility function $L$ times the pressure difference:

$$
\frac{\mathrm{d} \epsilon}{\mathrm{d} t}=L \Delta p=q \epsilon
$$

Using the results of Tomotika for the growth rate in the absence of a centrifugal field (eq 4), we find for the mobility function

$$
L=\frac{R_{0} \Phi\left(\eta_{1} / \eta_{2}, X\right)}{4 \eta_{2}}
$$

Now, assume that in the final stage there is a finite rotational speed causing a centrifugal field. The effect of such a centrifugal field on the stability of a liquid thread surrounded by a denser liquid was already considered by Rosenthal ${ }^{21} 40$ years ago. He showed that the rotation has a stabilizing influence and shifts the wavelengths of the unstable disturbances to higher values. However, Rosenthal did not take into account the viscosities of the liquid thread and the surrounding denser liquid, and hence his results are not directly applicable to our experimental situation. Here we present a simple model which takes into account these viscosities. It yields an expression for the growth rates of the unstable disturbances and allows analysis of our experimental results.

The centrifugal field influences the pressure difference, which in turn effects the stability of the liquid thread. The contribution of the centrifugal field to the pressure difference can be determined as (which is clear by the analysis of the pressure difference along path $\mathrm{A}-\mathrm{C}$ compared to path $\mathrm{B}-\mathrm{D}$ in Figure 1 , assuming the pressure difference between points $\mathrm{C}$ and $\mathrm{D}$ is zero):

$$
\begin{aligned}
& \Delta p_{\text {centr }}=\frac{1}{2} \omega^{2} \rho_{1}\left(R_{0}+\epsilon\right)^{2}- \\
&\left\{\frac{1}{2} \omega^{2} \rho_{1}\left(R_{0}-\epsilon\right)^{2}+\frac{1}{2} \omega^{2} \rho_{2}\left[\left(R_{0}+\epsilon\right)^{2}-\left(R_{0}-\epsilon\right)^{2}\right]\right\}= \\
&-2 \epsilon \Delta \rho \omega^{2} R_{0}
\end{aligned}
$$

The total pressure difference will then be

$$
\Delta p=\Delta p_{\text {Laplace }}+\Delta p_{\text {centr }}=\frac{2 \epsilon}{R_{0}^{2}}\left[\gamma\left(1-X^{2}\right)-\Delta \rho \omega^{2} R_{0}^{3}\right]
$$

Finally, the combination of eq 9 and eq 12, and assuming the mobility function derived by Tomotika (eq 10) still to be valid in the presence of a centrifugal field, gives an expression for the growth rate of the breakup in a centrifugal field:

$$
q=\frac{\Phi\left(\eta_{1} / \eta_{2}, X\right)}{2 \eta_{2} R_{0}}\left[\gamma\left(1-X^{2}\right)-\Delta \rho \omega^{2} R_{0}^{3}\right]
$$

Equation 13 is the central result of our model, and it predicts that the liquid thread becomes unstable for

$$
X^{2}+\frac{\Delta \rho \omega^{2} R_{0}^{2}}{\gamma}<1
$$

This condition agrees completely with the stability criterion already obtained by Rosenthal. ${ }^{21}$ This is not surprising since as remarked by Batchelor (quoted in the paper by Rosenthal ${ }^{21}$ ) in the problem considered here, viscosity has no effect on the curve of neutral stability. However, viscosity will affect the growth rates of unstable disturbances.

Although the derivation of eq 13 can be physically motivated and is justified in part, its quantitative correctness can only be assessed by a full linear stability analysis of the problem. Here we will test eq 13 by comparing it to our experimental results. To do so, we plot $q$ as a function of $X$, using eq 13, for various rotational speeds (Figure 5). For the interfacial tension we used a value of $3.71 \mu \mathrm{N} / \mathrm{m}$, as measured with the spinning drop method for this specific sample. The viscosities of the two fluids $\left(\eta_{1}=8.4 \mathrm{mPa} \mathrm{s}, \eta_{2}=97.1 \mathrm{mPa} \mathrm{s}\right)$ and the radius of the droplet 


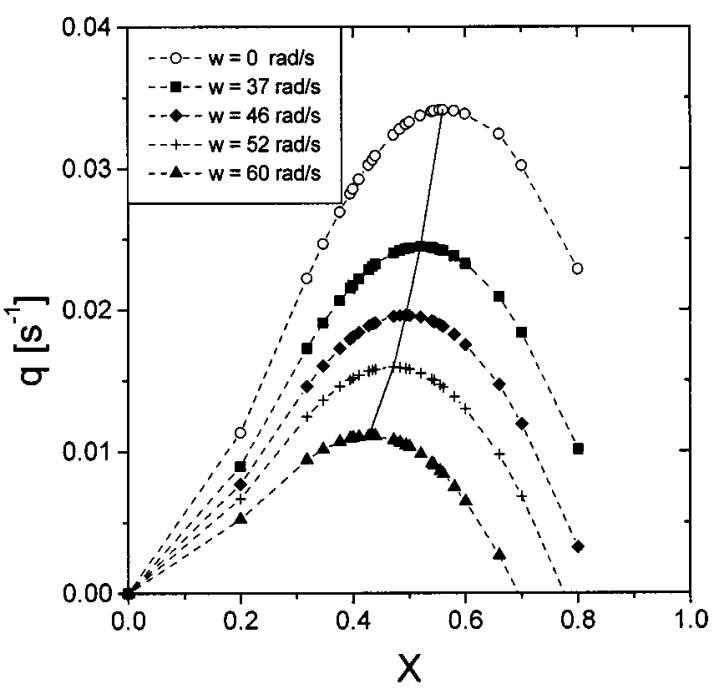

Figure 5. Growth rate, $q$, as a function of the reduced wavelength, $X$, for various rotational speeds calculated with eq 13 .
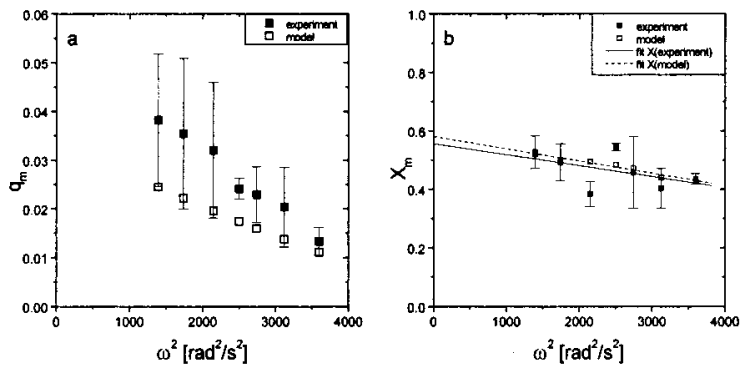

Figure 6. (a) Values for $q_{\mathrm{m}}$ as a function of $\omega^{2}$ obtained by experiment and the model. (b) Values for $X_{\mathrm{m}}$ as a function of $\omega^{2}$ obtained by experiment and the model. Both data sets were extrapolated to zero rotational speed.

as measured (typically $\sim 0.13 \mathrm{~mm}$ ) were used to be able to compare the calculated values with the experimental data. In the plot we identify the maximim of $q$ as $q_{\mathrm{m}}$ and that of the corresponding $X$ as $X_{\mathrm{m}}$. It is clear from Figure 5 that $q_{\mathrm{m}}$ and $X_{\mathrm{m}}$ depend on the final rotational speed. The calculated values are compared with the experimental values as a function of the final rotational speed squared, $\omega^{2}$, in Figure 6.

We find the same trend of $q_{\mathrm{m}}$ as a function of $\omega^{2}$ for the experimental and calculated data. The magnitude differs, although they are of the same order. The experimental values are about 1.5 times larger than the calculated ones. The experimental and calculated values of $X_{\mathrm{m}}$ are in good agreement. Extrapolation to zero rotational speed gives for the experimental data a value of 0.55 , and for the calculated values it is 0.58 . Both values are close to the value of 0.56 as calculated according to the analysis of Tomotika. The small difference between the experimental value and the one obtained following Tomotika justifies the smoothing of the experimental data with the fitted curve. These smoothed values are used in the following paragraph. From the comparison between the calculated and experimental results it appeared that the estimation of the influence of the centrifugal field on the growth rate of the disturbance of a droplet is reasonably described with this simple model.

Now we can use eq 13 the other way around: the determination of the interfacial tension from measured growth rates and wavelengths as a function of the rotational speed. Using measured values of $X_{\mathrm{m}}$ as a function of $\omega$, the effect of the centrifugal field on mobility function is accounted for to some extent. Figure 7 shows the results. For comparison we show

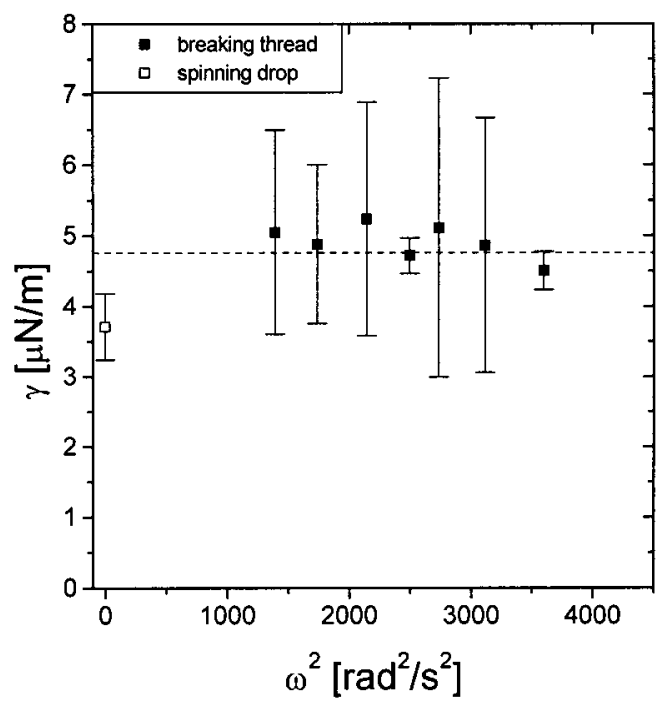

Figure 7. Interfacial tension, $\gamma$, calculated with eq 13 . The value measured with the spinning drop is also indicated.

also the interfacial tension of $3.71 \pm 0.47 \mu \mathrm{N} / \mathrm{m}$ found with the spinning drop method for this specific sample. A linear fit through the data gives an interfacial tension of $4.76 \pm 1.47$ $\mu \mathrm{N} / \mathrm{m}$, which is about $25 \%$ larger than the value measured with the spinning drop method. The difference is expected because of the discrepancy between the experimental and calculated values of $q_{\mathrm{m}}$. Furthermore, it is known that a dynamic interfacial tension is sometimes higher than a stationary interfacial tension.

\section{Conclusions}

We have shown that the rate of breakup of a droplet in a centrifugal field depends not only on the interfacial tension, but also on the magnitude of the centrifugal field applied. We found that the growth rate of the disturbance decreases and the wavelength of the disturbance increases with an increase of the centrifugal field. We developed a simple model to analyze the effect of the centrifugal field. This model is derived from a physical understanding of the stability of a liquid thread in a surrounding fluid, taking into account the viscosities of the two fluids. It yields an expression for the growth rate of the unstable disturbances. The calculated interfacial tension, determined by the measured growth rates and wavelengths and using this model, is in reasonable agreement with the value found with the stationary method of the spinning drop. The determination of a small interfacial tension between two fluids with low viscosities following the method described in this paper yields a considerable gain of time compared to the spinning drop technique.

Acknowledgment. The authors are grateful to Prof. Howard Stone for his encouragement and help to clarify the position of our work. This work was supported by the Stichting voor Fundamenteel Onderzoek der Materie (Foundation for Fundamental Research on Matter) which is part of the Nederlandse Organisatie voor Wetenschappelijk Onderzoek (Netherlands Organization for Advancement of Research).

\section{References and Notes}

(1) Stone, H. A. Annu. Rev. Fluid Mech. 1994, 26, 65.

(2) Elemans, P. H. M. Ph.D. Thesis, Technical University Eindhoven, Eindhoven, The Netherlands, 1989.

(3) Döring, M. Philips Technol. Rev. 1982, 40, 192.

(4) Han, C. D. Multiphase Flow in Polymer Processing; Academic: New York, 1981. 
(5) Tjahjadi, M.; Ottino, J. M.; Stone, H. A. AIChE J. 1994, 40, 385. (6) Elemans, P. H. M.; Janssen, J. M. H.; Meijer, H. E. H. J. Rheol. 1990, $34,1311$.

(7) Vliegenthart, G. A.; Lekkerkerker, H. N. W. Prog. Colloid Polym. Sci. 1997, 105, 27.

(8) de Hoog, E. H. A.; Lekkerkerker, H. N. W. J. Phys. Chem. B 1999, 103,5274 .

(9) Chen, B.-H.; Payandeh, B.; Robert, M. Phys. Rev. E 2000, 62, 2369.

(10) van der Schoot, P. J. Phys. Chem. B 1999, 103, 8804.

(11) Brader, J. M.; Evans, R. Europhys. Lett. 2000, 49, 678

(12) Brader, J. M.; Evans, R.; Schmidt, M.; Löwen, H. J. Phys. Condens. Matter, submitted.
(13) Vonnegut, B. Rev. Sci. Instrum. 1942, 13, 6.

(14) Princen, H. M.; Zia, I. Y. Z.; Mason, S. G. J. Colloid Interface Sci. 1967, 23, 99 .

(15) Rayleigh, Lord Proc. London Math. Soc. 1878, 10, 4

(16) Tomotika, S. Proc. R. Soc., Ser. A 1935, 150, 322.

(17) Rumscheidt, F. D.; Mason, S. G. J. Colloid Sci. 1962, 17, 260.

(18) Verhaegh, N. A. M.; van Duijneveldt, J. S.; Dhont, J. K. G.; Lekkerkerker, H. N. W. Physica A 1996, 230, 409.

(19) Bodnár, I.; Oosterbaan, W. D. J. Chem. Phys. 1997, 106, 7777. (20) van Aken, G. A. Ph.D. Thesis, Utrecht University, Utrecht, The Netherlands, 1990.

(21) Rosenthal, D. K. J. Fluid Mech. 1962, 12, 358 